\title{
Some Mathematical and Numerical Aspects in Aluminum Production
}

\author{
M. Flueck • A. Janka • C. Laurent • M. Picasso • \\ J. Rappaz • G. Steiner
}

Received: 5 October 2007 / Revised: 2 April 2008 / Accepted: 2 July 2008 / Published online: 23 July 2008 (C) Springer Science+Business Media, LLC 2008

\begin{abstract}
In this paper, we present a mathematical modeling of some magnetohydrodynamic effects arising in an aluminum production cell as well as its numerical approximation by a finite element method. We put the emphasis on the magnetic effects which live in the whole three dimensional space and which are solved numerically with a domain decomposition method.
\end{abstract}

Keywords Maxwell equations · Domain decomposition · Magnetohydrodynamics $\cdot$ Free surface problem $\cdot$ Aluminum production

\section{Introduction}

The manufacturing process in the aluminum production industry is to inject alumina $\mathrm{Al}_{2} \mathrm{O}_{3}$ in an electrolytic cell schematically represented in Fig. 1 in order to obtain the chemical reaction:

$$
2 \mathrm{Al}_{2} \mathrm{O}_{3}+\text { electric energy } \rightarrow 4 \mathrm{Al}+3 \mathrm{O}_{2} .
$$

The oxygen is then burnt by anodes:

$$
\mathrm{C}+\mathrm{O}_{2} \rightarrow \mathrm{CO}_{2}
$$

and the aluminum is collected at the cathodic bloc at the bottom of the cell. Two liquids are present in a cell. At the top, close to the anodes, there is the electrolytic bath in which the

This work is supported by Alcan Company, Laboratoire de Recherche et Fabrication, Saint Jean de Maurienne, France.

M. Flueck · A. Janka · C. Laurent · M. Picasso · J. Rappaz · G. Steiner ( $\varangle)$

Chair of Numerical Analysis and Simulation, Ecole Polytechnique Fédérale de Lausanne, Lausanne,

Switzerland

e-mail: gilles.steiner@epfl.ch

J. Rappaz

e-mail: jacques.rappaz@epfl.ch 
Fig. 1 Diagram of an electrolytic cell (Transverse section)

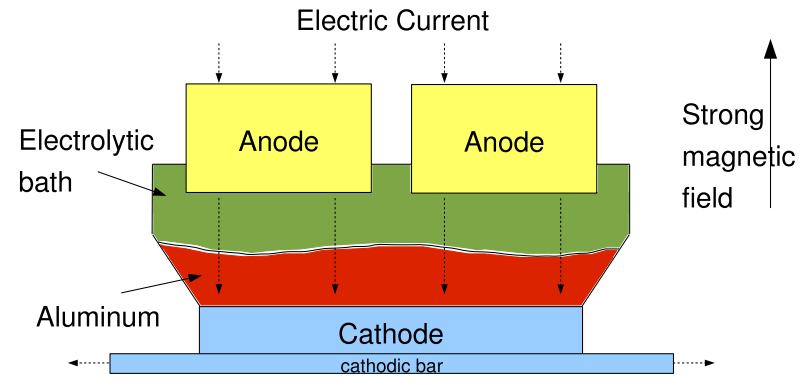

reaction occurs and, at the bottom, the liquid aluminum is deposited on the cathodic bloc. A very strong electric current density $\vec{j}$ flows through about a hundred electrolytic cells and conductors contained in the hall for aluminum production $\left(\sim 6000 \mathrm{~A} / \mathrm{m}^{2}\right.$ in a cell). This current produces a strong magnetic induction field $\vec{B}(\sim 1000$ Gauss). In particular in the cells, Lorentz forces $\vec{j} \wedge \vec{B}$ give rise to hydrodynamic effects and the velocity $\vec{u}$ of the fluids can reach $0.5 \mathrm{~m} / \mathrm{s}$. The stability of the bath-aluminum interface is very important in order to obtain a cell working in adequate conditions. Among the different approaches found in the literature in order to study this phenomenon, let us mention Fourier analysis of linear models (see for instance [1]), linear stability of MHD stationary solutions (see for instance [2, 3]), or numerical modeling of the time dependent MHD equations (see [4] and its references). In this paper we present a time dependent numerical model as in [4] and compute the induction magnetic field $\vec{B}$ together with the fluid motion.

For this purpose, we have to couple

- The incompressible Navier-Stokes equations with free surface to obtain the pressure $p$ and the velocity $\vec{u}$ from the gravity forces $\rho \vec{g}$ and the electric forces $\vec{j} \wedge \vec{B}$;

- An elliptic equation for the potential $V$ to obtain the electric current

$$
\vec{j}=\sigma\left(-\vec{\nabla} V-\frac{\partial \vec{A}}{\partial t}+\vec{u} \wedge \vec{B}\right),
$$

where $\sigma$ is the electric conductivity of materials and $\vec{A}$ the magnetic potential;

- Maxwell's equations to obtain $\vec{B}$ from the current density $\vec{j}$.

All these equations are solved numerically with finite elements methods in space.

Navier-Stokes equations and the bath-aluminum free surface are discretized in time with a splitting method of order two à la Strang-Glowinski [7] and by using a level set technique.

The fields $\vec{B}$ and $\vec{A}$ are obtained from a domain decomposition method (see $[10,12]$ ).

Numerical results on a parallelepipedic geometry will be presented in order to show the origins of instabilities in aluminum production cells.

\section{The Hydrodynamic Problem}

\subsection{Modeling}

In this section we denote by $\Omega$ the domain occupied by both liquid aluminum and electrolytic bath, by $\Omega_{a}(t)$ the domain occupied by the liquid aluminum and by $\Omega_{b}(t)$ the domain occupied by the electrolytic bath where $t$ is the time. Clearly we have $\bar{\Omega}=\bar{\Omega}_{a}(t) \cup \bar{\Omega}_{b}(t)$ and 
the interface between $\Omega_{a}(t)$ and $\Omega_{b}(t)$ will be denoted by $\Gamma(t)$ i.e. $\Gamma(t)=\bar{\Omega}_{a}(t) \cap \bar{\Omega}_{b}(t)$. We assume there exist a domain $\Lambda \subset \mathbb{R}^{2}$ and a smooth function $g:(0, \infty) \times \Lambda \rightarrow \mathbb{R}$ satisfying

$$
\Gamma(t)=\left\{(x, y, z) \in \mathbb{R}^{3}: z=g(t, x, y),(x, y) \in \Lambda, t \in(0, \infty)\right\},
$$

i.e. $\Gamma(t)$ can be parametrized with the variables $x$ and $y$. The aluminum has a constant density $\rho_{a}$ greater than the density of the bath $\rho_{b}$ and consequently is under $\Gamma(t)$ whereas the bath is above $\Gamma(t)$. The viscosities of aluminum and bath are denoted by $\mu_{a}$ and $\mu_{b}$ respectively and are assumed to be constant. In order to simplify the notations, we will write in the following only $\rho$ or $\mu$ when there is no ambiguity. Assume that current density $\vec{j}$ and the induction magnetic field $\vec{B}$ are known. The forces $\vec{f}$ having effect on both fluids are given by $\vec{f}=\rho \vec{g}+\vec{j} \wedge \vec{B}$ and the velocity $\vec{u}$ and pressure $p$ satisfy the incompressible Navier-Stokes equations:

$$
\left\{\begin{array}{l}
\rho \frac{\partial \vec{u}}{\partial t}+\rho(\vec{u} \cdot \vec{\nabla}) \vec{u}-\operatorname{div}(\tau(\vec{u}, p))=\vec{f}, \\
\operatorname{div}(\vec{u})=0,
\end{array}\right.
$$

where the stress tensor $\boldsymbol{\tau}$ is defined by

$$
\boldsymbol{\tau}(\vec{u}, p)=2 \mu \boldsymbol{\varepsilon}(\vec{u})-p I, \quad \text { with } \boldsymbol{\varepsilon}(\vec{u})=\frac{1}{2}\left(\vec{\nabla} \vec{u}+\vec{\nabla} \vec{u}^{T}\right) .
$$

If $\vec{n}$ is the unit normal vector on $\Gamma(t)$ pointing to $\Omega_{b}(t)$, we assume that the velocity $\vec{u}$ and the force $\tau(\vec{u}, p) \cdot \vec{n}$ are continuous through the interface $\Gamma(t)$ and we write $[\vec{u}]=0$ and $[\tau(\vec{u}, p) \cdot \vec{n}]=0$ where $[\beta]=\beta_{b}-\beta_{a}$ is the jump of the variable $\beta$ between $\Omega_{a}(t)$ and $\Omega_{b}(t)$. Remark that we can neglect surface electromagnetic and surface tension effects, due to the large ratio between the characteristic dimensions of the cells $(\sim 5-10 \mathrm{~m})$ against the capillary length $(\sim 2 \mathrm{~cm})$. Considering slip conditions on the boundary $\partial \Omega$ of $\Omega$, a classical weak formulation of the above problem becomes: find two mappings $\vec{u}: t \in(0, \infty) \rightarrow \vec{u}(t) \in$ $\widetilde{H}^{1}(\Omega)$ and $p: t \in(0, \infty) \rightarrow p(t) \in L_{0}^{2}(\Omega)$ satisfying

$$
\begin{aligned}
\int_{\Omega}\left(\rho \frac{\partial \vec{u}}{\partial t} \cdot \vec{v}+\rho(\vec{u} \cdot \vec{\nabla}) \vec{u} \cdot \vec{v}+\boldsymbol{\tau}(\vec{u}, p): \boldsymbol{\varepsilon}(\vec{v})\right) d \Omega & =\int_{\Omega} \vec{f} \cdot \vec{v} d \Omega, \\
\int_{\Omega} q \operatorname{div}(\vec{u}) d \Omega & =0,
\end{aligned}
$$

for all $\vec{v} \in \widetilde{H}^{1}(\Omega)=\left\{\vec{v} \in H^{1}(\Omega)^{3}\right.$ satisfying $\vec{v} \cdot \vec{n}=0$ on $\left.\partial \Omega\right\}$, and all $q \in L_{0}^{2}(\Omega)=\{q \in$ $\left.L^{2}(\Omega): \int_{\Omega} q d x=0\right\}\left(L^{2}(\Omega)\right.$ being the standard space of square integrable functions and $H^{1}(\Omega)$ the standard Sobolev space). We refer to [6] and [7] for the discussion on the existence of the solution.

If $\varphi:(0, \infty) \times \Omega \rightarrow \mathbb{R}$ is a smooth function, negative in $\Omega_{a}(t)$ and positive in $\Omega_{b}(t)$, we can also describe $\Gamma(t)$ by $\Gamma(t)=\{(x, y, z) \in \Omega: \varphi(t, x, y, z)=0\}$ and we have the transport equation

$$
\frac{\partial \varphi}{\partial t}+\vec{u} \cdot \vec{\nabla} \varphi=0 \text { in } \Omega
$$

with the relationships

$$
\begin{aligned}
& \Omega_{a}(t)=\{(x, y, z) \in \Omega: \varphi(t, x, y, z)<0\}, \\
& \Omega_{b}(t)=\{(x, y, z) \in \Omega: \varphi(t, x, y, z)>0\} .
\end{aligned}
$$




\subsection{Numerical Scheme in Time}

For solving the above problem we use a second order splitting method in time à la StrangGlowinski (see [7]). Given the time discretization

$$
0<t_{1}<t_{2}<\cdots<t_{n}<t_{n+1}<\cdots, \quad \text { with } t_{n+\frac{1}{2}}=\frac{t_{n}+t_{n+1}}{2},
$$

if $\vec{u}=\vec{u}^{n}, p=p^{n}$ and $\varphi=\varphi^{n}$ are known at time $t_{n}$, we compute $\vec{u}^{n+1}, p^{n+1}$ and $\varphi^{n+1}$ at time $t_{n+1}$ by:

- Stokes equation on $\left(t_{n}, t_{n+\frac{1}{2}}\right)$,

$$
\begin{array}{ll}
\int_{\Omega}\left(\rho \frac{\partial \vec{u}}{\partial t} \cdot \vec{v}+\tau(\vec{u}, p): \varepsilon(\vec{v})\right) d \Omega=\int_{\Omega} \vec{f} \cdot \vec{v} d \Omega, & \forall \vec{v} \in \widetilde{H}^{1}(\Omega), \\
\int_{\Omega} q \operatorname{div}(\vec{u}) d \Omega=0, & \forall q \in L_{0}^{2}(\Omega), \\
\vec{u}\left(t_{n}\right)=\vec{u}^{n} . &
\end{array}
$$

- Transport equation on $\left(0, \Delta t_{n}\right)$, with $\Delta t_{n}=t_{n+1}-t_{n}$, and $\vec{u}^{n+\frac{1}{2}}=\vec{u}\left(t_{n+\frac{1}{2}}\right)$ as advection field,

$$
\begin{aligned}
& \frac{\partial \vec{u}}{\partial t}+\left(\vec{u}^{n+\frac{1}{2}} \cdot \vec{\nabla}\right) \vec{u}=0, \\
& \frac{\partial \varphi}{\partial t}+\vec{u}^{n+\frac{1}{2}} \cdot \vec{\nabla} \varphi=0, \\
& \vec{u}(0)=\vec{u}^{n+\frac{1}{2}}, \varphi(0)=\varphi^{n} .
\end{aligned}
$$

- Stokes equation on $\left(t_{n+\frac{1}{2}}, t_{n+1}\right)$,

$$
\begin{array}{ll}
\int_{\Omega}\left(\rho \frac{\partial \vec{u}}{\partial t} \cdot \vec{v}+\boldsymbol{\tau}(\vec{u}, p): \boldsymbol{\varepsilon}(\vec{v})\right) d \Omega=\int_{\Omega} \vec{f} \cdot \vec{v} d \Omega, & \forall \vec{v} \in \widetilde{H}^{1}(\Omega), \\
\int_{\Omega} q \operatorname{div}(\vec{u}) d \Omega=0, & \forall q \in L_{0}^{2}(\Omega), \\
\vec{u}\left(t_{n+\frac{1}{2}}\right)=\vec{u}\left(\Delta t_{n}\right) . &
\end{array}
$$

We set $\vec{u}^{n+1}=\vec{u}\left(t_{n+1}\right), p^{n+1}=p\left(t_{n+1}\right)$ and $\varphi^{n+1}=\varphi\left(\Delta t_{n}\right)$. Both Stokes problems are solved by an implicit Runge-Kutta method in time and by a P1-P1 FEM with stabilization for the space discretization (see [9]). Both transport equations are solved by using a "wavelike formulation" à la Glowinski (see [7, 8]) with a Newmark method for the discretization in time, and with a P1-FEM for the space discretization. All these problems are numerically solved on the same tetrahedral mesh $\mathcal{T}_{h}^{n}$ of size $h$ which covers $\bar{\Omega}$. The linear systems are solved by CG or GMRES methods.

In order to compute the evolution of the physical variables (electric current density, magnetic induction, etc.) on the interface, an easy method is to move the mesh according to the displacement of the interface since the topology of the fluids domains does not change. In 
this case we could use either the ALE method or an interpolation method. In our computations, these two methods have been tested and give rise to similar results, but since in both cases we need to interpolate the initial magnetic induction field and current density, we have chosen to use the interpolation method described below.

The two dimensional domain $\bar{\Lambda}$ is discretized with a triangular mesh $\mathcal{G}_{h}$ and all the vertices of $\mathcal{T}_{h}^{n}$ are moved along prescribed segments going through the vertices of $\mathcal{G}_{h}$. The interface $\Gamma$ at time $t_{n}$ is denoted by $\Gamma^{n}$ and is made with triangular faces of $\mathcal{T}_{h}^{n}$. When the above time step is finished, the nodes of $\Gamma^{n}$ are moved on these lines in such a way that $\varphi^{n+1}$ is vanishing in order to build $\Gamma^{n+1}$. All the mesh $\mathcal{T}_{h}^{n}$ is stretched and adapted to $\Gamma^{n+1}$ to give rise to the new mesh $\mathcal{T}_{h}^{n+1}$ on which the velocity, initial magnetic induction and electric current density fields are interpolated. A smoothing procedure is used to avoid the numerical instabilities that could appear on the interface. It is based on the approximation of the function describing the interface with a $2 \mathrm{D}$ polynomial that minimize the distance to the interface in a least square sense.

\section{The Electric Potential and Current Density}

\subsection{Modeling}

In this paragraph we consider the whole aluminum cell $\Sigma$ which is the union of conductors (anodes, cathodic bars) and both fluids in $\Omega$ (see Fig. 1). We assume that at time $t$ the velocity of the fluids $\vec{u}$ and the magnetic induction field $\vec{B}$ are known and we want to compute the current density $\vec{j}$.

If $\vec{E}$ is the electric field, Faraday's law is the following

$$
\frac{\partial \vec{B}}{\partial t}+\operatorname{curl}(\vec{E})=0 \text { in all the space } \mathbb{R}^{3} .
$$

Using Gauss' law $\operatorname{div} \vec{B}=0$, we can ensure that there exists a vector potential $\vec{A}$ defined in all the space $\mathbb{R}^{3}$ satisfying

$$
\vec{B}=\operatorname{curl}(\vec{A}),
$$

with Coulomb's gauge $\operatorname{div} \vec{A}=0$. It follows that

$$
\operatorname{curl}\left(\vec{E}+\frac{\partial \vec{A}}{\partial t}\right)=0,
$$

and there exists a scalar potential $V$ such that

$$
\vec{E}+\frac{\partial \vec{A}}{\partial t}=-\vec{\nabla} V
$$

In the cell $\Sigma$ we assume that the Ohm's law is valid

$$
\vec{j}=\sigma(\vec{E}+\vec{u} \wedge \vec{B})=\sigma\left(-\frac{\partial \vec{A}}{\partial t}-\vec{\nabla} V+\vec{u} \wedge \vec{B}\right),
$$

where $\vec{u}=0$ in $\Sigma \backslash \bar{\Omega}$ and $\sigma$ is the electric conductivity of various materials bringing the current in the cell. Moreover, if we neglect the displacement current in Ampere's law (see [13], 
pp. 38-40, for a justification), we have

$$
\operatorname{curl}(\vec{B})=\mu_{0} \vec{j}
$$

where $\mu_{0}$ is the magnetic permeability of the void, and consequently

$$
\operatorname{div} \vec{j}=0 .
$$

It follows from (19) and (21) that

$$
-\operatorname{div}(\sigma \vec{\nabla} V)=-\operatorname{div}\left(\sigma\left(-\frac{\partial \vec{A}}{\partial t}+\vec{u} \wedge \vec{B}\right)\right) \quad \text { in } \Sigma .
$$

Remark that outside $\Omega$, we have $\vec{u}=0$ and consequently

$$
-\operatorname{div}(\sigma \vec{\nabla} V)=\operatorname{div}\left(\sigma \frac{\partial \vec{A}}{\partial t}\right) \quad \text { in } \Sigma \backslash \bar{\Omega} .
$$

The electric current going in $\Sigma$ through the anodic bars is imposed on an input surface $S_{i} \subset \partial \Sigma$, i.e.

$$
\vec{j}_{i} \cdot \vec{n}=-\sigma \frac{\partial V}{\partial n}-\sigma \frac{\partial \vec{A}}{\partial t} \cdot \vec{n}, \quad \text { on } S_{i},
$$

with $\vec{n}$ being the outgoing unit normal vector. Similarly, the electric current going out $\Sigma$ through the cathodic bars is given and imposed on $S_{o} \subset \partial \Sigma$, i.e.

$$
\vec{j}_{o} \cdot \vec{n}=-\sigma \frac{\partial V}{\partial n}-\sigma \frac{\partial \vec{A}}{\partial t} \cdot \vec{n}, \quad \text { on } S_{o} .
$$

On the rest of the boundary $\partial \Sigma$ we set

$$
\vec{j} \cdot \vec{n}=-\sigma \frac{\partial V}{\partial n}-\sigma \frac{\partial \vec{A}}{\partial t} \cdot \vec{n}=0, \quad \text { on } \partial \Sigma \backslash\left(S_{i} \cup S_{o}\right) .
$$

Assume that $\vec{A}$ is known (the method will be described in the next part), (22) can then be used to compute $V$, provided boundary conditions (24)-(26) are prescribed. We are thus looking for a potential $V \in H^{1}(\Sigma)$ such that

$$
\begin{aligned}
\int_{\Sigma} \sigma \vec{\nabla} V \cdot \vec{\nabla} W d \Omega= & -\int_{\Sigma} \sigma \frac{\partial \vec{A}}{\partial t} \cdot \vec{\nabla} W d \Omega+\int_{\Omega} \sigma(\vec{u} \wedge \vec{B}) \cdot \vec{\nabla} W d \Omega \\
& -\int_{S_{i}}\left(\overrightarrow{j_{i}} \cdot \vec{n}\right) W d S-\int_{S_{o}}\left(\vec{j}_{o} \cdot \vec{n}\right) W d S
\end{aligned}
$$

for all $W \in H^{1}(\Sigma)$. Once $V$ is known, we can obtain the current density by the formula (19).

\subsection{Numerical Scheme}

In order to obtain an approximation $V_{h}$ of $V$, we proceed with a standard finite element method of degree 1, i.e. if $H_{h}^{1} \subset H^{1}(\Sigma)$ is a finite dimensional space built with piecewise 
polynomial functions of degree 1 on a tetrahedral mesh $\Sigma_{h}$ discretizing $\Sigma$, we are looking for $V_{h} \in H_{h}^{1}$ satisfying

$$
\begin{aligned}
\int_{\Sigma} \sigma \vec{\nabla} V_{h} \cdot \vec{\nabla} W_{h} d \Omega= & -\int_{\Sigma} \sigma \frac{\partial \vec{A}}{\partial t} \cdot \vec{\nabla} W_{h} d \Omega+\int_{\Omega} \sigma(\vec{u} \wedge \vec{B}) \cdot \vec{\nabla} W_{h} d \Omega \\
& -\int_{S_{i}}\left(\overrightarrow{j_{i}} \cdot \vec{n}\right) W_{h} d S-\int_{S_{o}}\left(\overrightarrow{j_{o}} \cdot \vec{n}\right) W_{h} d S
\end{aligned}
$$

for all $W_{h} \in H_{h}^{1}$.

\section{The Magnetic Induction Field}

\subsection{Modeling}

Let us suppose now that the current density $\vec{j}$ is known and let us show how it is possible to obtain the magnetic induction field $\vec{B}$ and the vector potential $\vec{A}$ from $\vec{j}$. It is well known that, in absence of ferromagnetic materials, the Biot-Savart's law holds, i.e.

$$
\vec{B}(P)=\frac{\mu_{0}}{4 \pi} \int_{\mathbb{R}^{3}} \vec{j}(Q) \wedge \frac{\overrightarrow{P Q}}{\| \overrightarrow{P Q \|^{3}}} d \Omega_{Q} \quad \text { for all } P \in \mathbb{R}^{3},
$$

and

$$
\vec{A}(P)=\frac{\mu_{0}}{4 \pi} \int_{\mathbb{R}^{3}} \vec{j}(Q) \frac{1}{\| \overrightarrow{P Q \|}} d \Omega_{Q} \quad \text { for all } P \in \mathbb{R}^{3} .
$$

Numerically, the computation of these integrals is time consuming and, since they must be computed at each time step, the above formulation has to be improved. To do this, we first remark that the electric current flows in the domain $\Sigma$, where we want to compute $\vec{B}$ and $\vec{A}$, and also in conductors outside $\Sigma$. At time $t=0$ we have no other choice to compute $\vec{B}$ and $\vec{A}$ than using the above Biot-Savart's formulas. Let us suppose that we have computed $\vec{B}$ and $\vec{A}$ at time $t=0$ and let us denote by $\vec{B}_{0}$ and $\vec{A}_{0}$ these two fields. In the following we show how it is possible to compute $\delta \vec{B}$ and $\delta \vec{A}$ with a very cheap numerical method in order to obtain $\vec{B}=\vec{B}_{0}+\delta \vec{B}$ and $\vec{A}=\vec{A}_{0}+\delta \vec{A}$ at each time step.

Outside the domain $\Sigma$ we assume that the electric current is not modified by the motions of both fluids inside $\Omega$ and at every time step we have $\vec{j}=\vec{j}_{0}+\delta \vec{j}$ where the current density $\vec{j}_{0}$ is the source of $\vec{B}_{0}$ and $\vec{A}_{0}$ and $\delta \vec{j}$ is the perturbation of $\vec{j}_{0}$ due to the motions of the fluids in $\Omega$. Clearly speaking, the support of $\delta \vec{j}$, denoted by $\operatorname{supp}(\delta \vec{j})$, is included in $\Sigma$. Using Ampere's and Gauss laws we have

$$
\begin{cases}\operatorname{curl} \delta \vec{B}=\mu_{0} \delta \vec{j}, & \text { in } \mathbb{R}^{3} \text { with } \operatorname{supp}(\delta \vec{j}) \subset \Sigma, \\ \operatorname{div} \delta \vec{B}=0, & \text { in } \mathbb{R}^{3} .\end{cases}
$$

From these relationships we obtain $\delta \vec{B}=\operatorname{curl} \delta \vec{A}$, with $\operatorname{div} \delta \vec{A}=0$, and

$$
\operatorname{curl}(\operatorname{curl} \delta \vec{A})=\mu_{0} \delta \vec{j}, \quad \text { in } \mathbb{R}^{3} \text { with } \operatorname{supp}(\delta \vec{j}) \subset \Sigma .
$$

It follows from these relations that

$$
-\Delta(\delta \vec{A})=\mu_{0} \delta \vec{j} \quad \text { in all } \mathbb{R}^{3},
$$


and we impose the following behaviour at infinity

$$
\|\delta \vec{A}(P)\|=\mathcal{O}\left(\|\overrightarrow{O P}\|^{-1}\right), \quad \text { when }\|\overrightarrow{O P}\| \rightarrow \infty .
$$

By setting $\Psi$ one of the components of $\delta \vec{A}$ and $f$ the corresponding component of $\mu_{0} \delta \vec{j}$, we have to solve the following problem:

Find $\Psi: \mathbb{R}^{3} \rightarrow \mathbb{R}$ satisfying

$$
-\Delta \Psi=f,
$$

with $|\Psi(x, y, z)|=\mathcal{O}\left(\left(x^{2}+y^{2}+z^{2}\right)^{-1 / 2}\right)$ when $\left(x^{2}+y^{2}+z^{2}\right) \rightarrow \infty$, and where $\operatorname{supp}(f)$ is compact.

\subsection{Numerical Scheme}

The fields $\vec{B}_{0}$ and $\vec{A}_{0}$ are computed at time $t=0$ using Biot-Savart's law and Gauss quadrature formulas. On the contrary, in order to compute $\delta \vec{B}$ and $\delta \vec{A}$ at each time step, we have to numerically solve three problems (for the three components of $\delta \vec{A}$ ) as in (35). To do this, we use a domain decomposition method together with a Poisson's formula for harmonic functions. We now present this technique in order to find an approximation of $\Psi$.

If $B_{r}$ is a ball with radius $r$ centered at the origin and containing the support of $f$, then $\Psi$ is harmonic outside $B_{r}$ and we have the well known Poisson's formula:

$$
\Psi(\vec{x})=\frac{\|\vec{x}\|^{2}-r^{2}}{4 \pi r} \int_{\partial B_{r}} \frac{\Psi(\vec{y})}{\|\vec{x}-\vec{y}\|^{3}} d S(\vec{y}), \quad \text { for all } \vec{x} \notin B_{r} .
$$

In particular if $B_{R}$ is a ball with radius $R>r$ centered at the origin, we have

$$
\begin{cases}-\Delta \Psi=f, & \text { in } B_{R}, \\ \Psi(\vec{x})=\frac{R^{2}-r^{2}}{4 \pi r} \int_{\partial B_{r}} \frac{\Psi(\vec{y})}{\|\vec{x}-\vec{y}\|^{3}} d S(\vec{y}), & \text { for all } \vec{x} \text { on } \partial B_{R} .\end{cases}
$$

Let us initialize the algorithm by $\Psi_{0}: B_{R} \rightarrow \mathbb{R}$, satisfying

$$
\begin{cases}-\Delta \Psi_{0}=f, & \text { in } B_{R}, \\ \Psi_{0}=0, & \text { on } \partial B_{R} .\end{cases}
$$

If $\Psi_{n}: B_{R} \rightarrow \mathbb{R}$ is known, we can compute $\Psi_{n+1}$ on $\partial B_{R}$ by:

$$
\Psi_{n+1}(\vec{x})=\frac{R^{2}-r^{2}}{4 \pi r} \int_{\partial B_{r}} \frac{\Psi_{n}(\vec{y})}{\|\vec{x}-\vec{y}\|^{3}} d S(\vec{y}), \quad \text { for all } \vec{x} \in \partial B_{R} .
$$

Using (38) as a Dirichlet boundary condition, we can find $\Psi_{n+1}$ in the interior of $B_{R}$, solving the problem

$$
-\Delta \Psi_{n+1}=f, \quad \text { in } B_{R} .
$$

Equation (39) is written in a weak form: Find $\Psi_{n+1} \in H^{1}\left(B_{R}\right)$ satisfying (38) on $\partial B_{R}$ and

$$
\int_{B_{R}} \vec{\nabla} \Psi_{n+1} \cdot \vec{\nabla} \phi d \Omega=\int_{B_{R}} f \phi d \Omega, \quad \text { for all } \phi \in H_{0}^{1}\left(B_{R}\right),
$$


where $H_{0}^{1}\left(B_{R}\right)$ is the standard Sobolev space of functions in $H^{1}\left(B_{R}\right)$ with vanishing trace on $\partial B_{R}$. This weak formulation is discretized by a finite element method of degree 1 and numerically solved using an algebraic multigrid method (AMG) which is very efficient (see [15]). The boundary condition (38), $\Psi_{n+1}(\vec{x})$ with $\vec{x} \in \partial B_{R}$, is computed by Gauss integration on the triangles discretizing $\partial B_{r}$. From the practical point of view, given a tetrahedral mesh of $\Sigma$, we have to generate a mesh of $B_{r} \backslash \Sigma$ which is compatible with $\Sigma$. In practice we use the GHS3D mesh generator (see [16] and [17]) which produces, given the surface meshes of $\partial \Sigma$ and of $\partial B_{r}$ a volume mesh of $B_{r} \backslash \Sigma$. Then, given the surface meshes of $\partial B_{r}$ and of $\partial B_{R}$, the volume mesh of $B_{R} \backslash B_{r}$ is constructed.

Remark that this method is in fact a Dirichlet-Dirichlet Schwarz algorithm with overlap $B_{R} \backslash \overline{B_{r}}$ since, in a first time we solve a harmonic problem outside $B_{r}$ and in a second time we solve a Dirichlet problem inside $B_{R}$, each time by taking boundary conditions coming from the previous problem.

Concerning this algorithm, it is easy to prove the following result (see [10]):

Theorem 4.1 We assume that $\operatorname{supp}(f) \subset B_{r}$ and that $f \in L^{\infty}\left(B_{r}\right)$. Then if $\Psi$ is the exact solution of the problem (35), we have the error estimate:

$$
\left\|\Psi-\Psi_{n}\right\|_{L^{\infty}\left(B_{R}\right)} \leq\left(\frac{r}{R}\right)^{n}\|\Psi\|_{L^{\infty}\left(\partial B_{R}\right)} .
$$

Note that this method can be extended to the computation of the screen effect of the steel ferromagnetic shell (see [12]).

\section{Algorithm and Numerical Results}

\subsection{Algorithm}

The time dependent problem modeling the magnetohydrodynamic effects in an aluminum production cell is numerically solved with a splitting method in time in which we compute successively the electric potential and the current density in all the cell and then the magnetic fields and the hydrodynamic forces arising in the liquid part of the cell. The algorithm is summarized by:

- Initialization:

At time $t=0$ the position of the aluminum-bath interface $\varphi$, the velocity $\vec{u}$ and the current density $\vec{j}_{0}$ are given. By using the Biot-Savart's law (see equations (29), (30)) the fields $\vec{A}_{0}, \vec{B}_{0}$ are computed. (At time $t=0$ we set $\frac{\partial \vec{A}}{\partial t}=0$ )

- For times $0<t_{1}<t_{2}<\cdots<t_{k}$ compute

1. The potential $V$ and the current density $\vec{j}$ using the techniques described in Sect. 3 . Set $\delta \vec{j}=\vec{j}-\overrightarrow{j_{0}}$.

2. The field $\delta \vec{A}$ with the domain decomposition method described in Sect. 4. Set $\vec{A}=$ $\vec{A}_{0}+\delta \vec{A}, \vec{B}=\operatorname{curl}(\vec{A}), \vec{f}=\rho \vec{g}+\vec{j} \wedge \vec{B}$.

3. The fields $\vec{u}$ and $p$ and the aluminum-bath interface $\varphi$ described in Sect. 2.

4. Go to next time step by returning to 1 . 
Fig. 2 The metal pad rolling in a simplified situation

Time $=0.100$

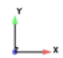

Time $=10.600$

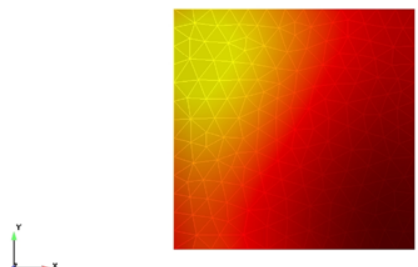

Time $=37.100$
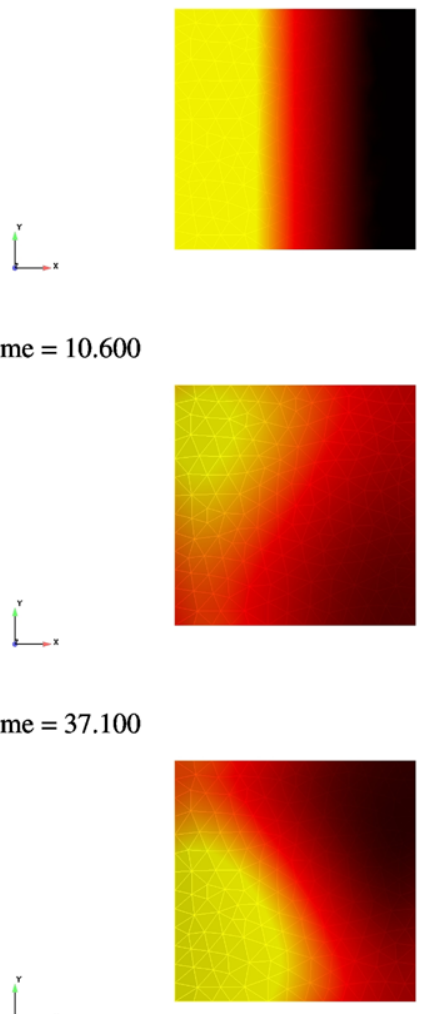

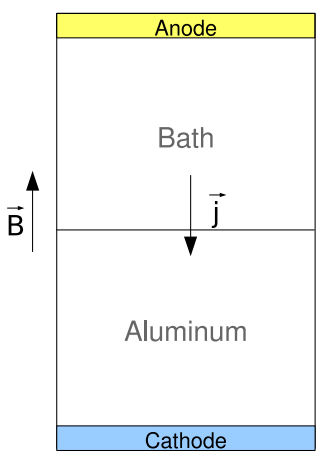

$\vec{F}=\vec{j} \wedge \vec{B}=0$

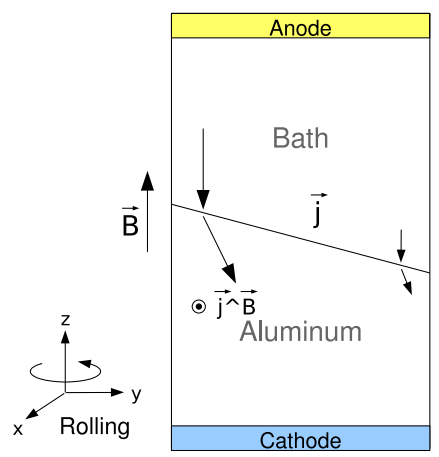

$\vec{F}=\vec{j} \wedge \vec{B} \neq 0$
Altitude [m]
$4.000 \mathrm{e}-03$
$2.000 \mathrm{e}-03$
$0.000 \mathrm{e}+00$
$-2.000 \mathrm{e}-03$
$-4.000 \mathrm{c}-03$

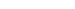

Time $=20.600$

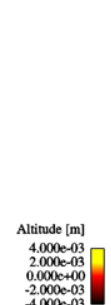

Time $=49.500$

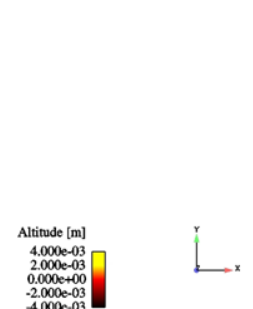

Altitude [m] $4.000 \mathrm{e}-03$
$2.000 \mathrm{e}-03$ $2.0000 \mathrm{e}-03$
$-2.000 \mathrm{e}-03$ $-2.000 \mathrm{e}-03$
$-4.000 \mathrm{e}-03$
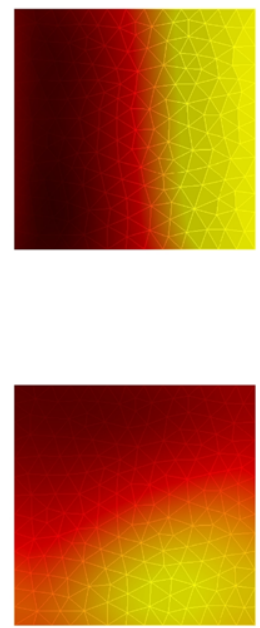

Altitude [m] $4.000 \mathrm{e}-03$
$2.000 \mathrm{e}-03$
$0.000 \mathrm{c}-00$ $0.0000+00$ $-2.0000-03$
$-4.0000-03$

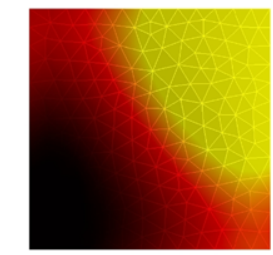

Altitude [m] $4,000 \mathrm{e}-03$
$2.000 \mathrm{e}-03$
$0.000 \mathrm{e}+00$ $0.000 \mathrm{e}+00$
$-2.000 \mathrm{e}-03$ $-2.000 \mathrm{e}-03$
$-4.000 \mathrm{e}-03$

Fig. 3 Rotational motion of the interface induced by Lorentz forces ( $x y$-view)

\subsection{Numerical Results}

In this section we present the numerical study of a typical phenomenon appearing in aluminum production: the metal pad rolling (Introduced by Sele in 1970 see [14], physically 


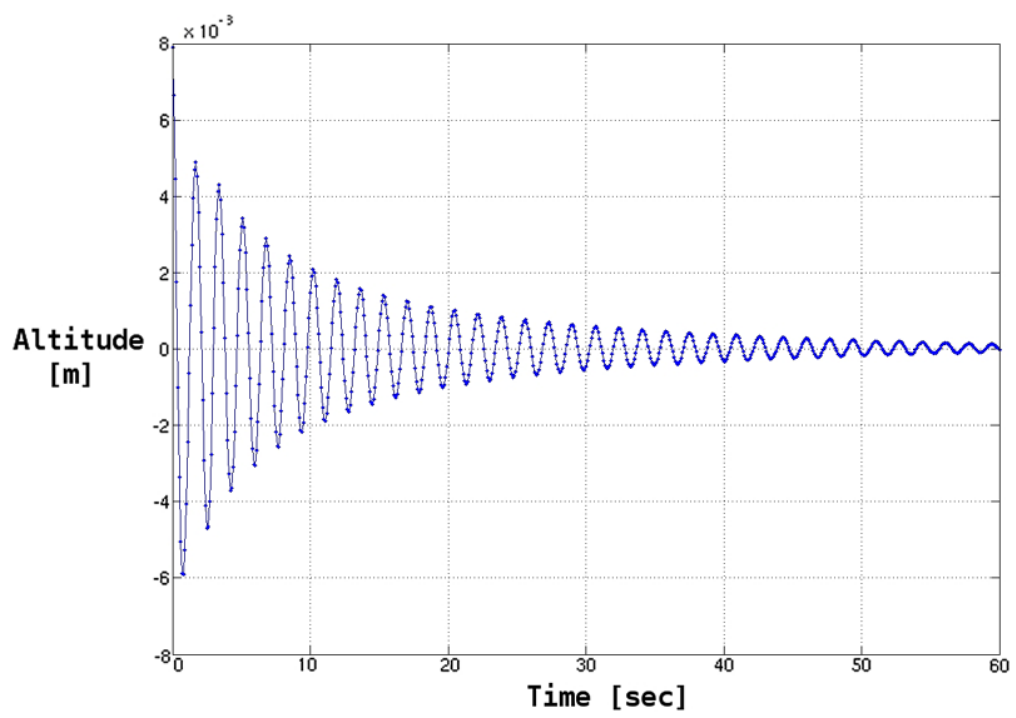

Fig. 4 Oscillation of the interface in a stable case

studied in [11] and numerically studied by Gerbeau et al. on a cylinder see [4] and [5]). Let us consider a simplified parallelepipedic electrolytic cell with both anode and cathode completely covering the top and bottom surfaces of the cell.

On the one hand, if the interface between aluminum and bath is horizontal, the current density $\vec{j}$ is perfectly vertical (Fig. 2). If the induction field $\vec{B}$ induced by exterior conductors and neighbours cells is vertical too the Lorentz forces $\vec{F}=\vec{j} \wedge \vec{B}$ is zero, and the fluids are static $(\vec{u}=0$ and interface is horizontal).

On the other hand, if the interface is perturbed, inclined like in the Fig. 2 for instance, due to the fact that the conductivity of aluminum is approximatively 15000 times bigger than the conductivity of the bath, the current density is no more uniform in the cell. In the neighbourhood of the interface, strong horizontal components of the current combined with the vertical component of the induction field $\vec{B}$ induce orthogonal Lorentz forces and a rotational motion of the fluids. In Fig. 3 we represent the evolution of the interface between the fluids in a parallelipipedic domain. We are looking at the interface in the $x y$-plane and the colors indicate the altitude of the nodes ( $z$ component of the position).

If the current density in the cell is smaller than a critical value, due to the viscosity of the fluids, the interface will reach the static state. In Fig. 4 we observe the oscillations of a point of the interface close to the border of the domain, starting from an inclined initial position. In this situation the cell is said to be stable as any reasonable perturbed initial state will come back to the steady state.

If the current density is too large, the aluminum-bath interface can oscillate with large amplitudes (cf. Fig. 5). In this case the cell is said to be unstable because a small perturbation of the steady state can produce a large motion of the interface, that can oscillate indefinitely.

\section{Conclusion}

The algorithms presented in this paper (and the one presented in [3]) has been implemented in a 3D Finite Element code named ALUCELL. It is used at an industrial level to simulate 


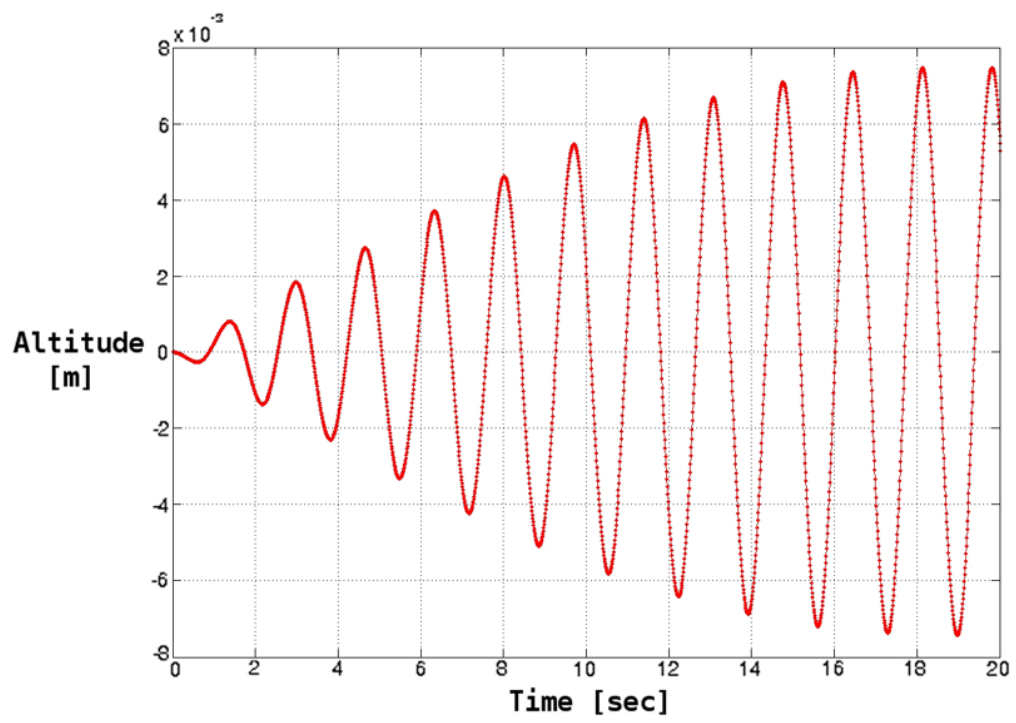

Fig. 5 Oscillation of the interface in an unstable case

the motion and stability of electrolytic cells and by the way, optimize the development of new technologies.

Acknowledgements The authors are very grateful to Aluminium-Péchiney Company for supporting this work.

\section{References}

1. Moreau, R., Ziegler, D.: Stability of aluminum cells, a new approach, Light Metals, 359-364 (1986)

2. Descloux, J., Flueck, M., Romerio, M.V.: Spectral aspects of an industrial problem. In: Spectral Analysis of Complex Structures, pp. 17-33. Hermann éditeurs des sciences et des arts, Paris (1995)

3. Descloux, J., Flueck, M., Romerio, M.V.: A modelling of the stability of aluminum electrolysis cells. In: Cioranescu, D., Lions, J.L. (eds.) Nonlinear partial differential equations and their applications. Collège de France Seminars, Vol. XIII Pitman Research Notes in Mathematics Series, vol. 391, pp. 117-133. Addison Wesley, Longman (1998)

4. Gerbeau, J.-F., Le Bris, C., Lelièvre, T.: Mathematical Methods for the Magnetohydrodynamics of Liquid Metals. Numerical Mathematics and Scientific Cumputation Series. Oxford Science Publications, Oxford (2006)

5. Gerbeau, J.-F., Le Bris, C., Lelièvre, T.: Metal pad roll instabilities, proceeding of the 2002 TMS Annual Meeting and Exhibition. Light Metals 483-487 (2002)

6. Lions, P.L.: Mathematical Topics in Fluid Mechanics, vol. 1. Oxford University Press, Oxford (1996)

7. Glowinski, R.: Finite element methods for incompressible viscous flow. In: Ciarlet, P.G., Lions, J.L. (eds.) Handbook of Numerical Analysis, vol. IX, pp. 498-541. North-Holland, Elsevier (2003)

8. Dean, E.J., Glowinski, R., Kuo, Y.M., Nasser, G.: On the Discretization of Some Second Order. In: Balakrisknan, A.V. (ed.) Time Differential Equations Applications to Nonlinear Wave Problems. Computational Techniques in Identification and Control of Flexible Flight Structures, pp. 199-246. Optimization Software, Inc., Los Angeles (1990)

9. Picasso, M., Rappaz, J.: Stability of time-splitting schemes for the Stokes problem with stabilized finite elements. Numer. Methods Partial Differ. Equ. 17(6), 632-656 (2001)

10. Flueck, M., Rappaz, J., Steiner, G.: On a domain decomposition method for numerically solving a magnetic induction problem. Scientific report in Analysis and Numerical Analysis, EPFL 
11. Davidson, P.A., Lindsay, R.I.: Stability of interfacial waves in aluminium reduction cells. J. Fluid Mech. 362, 273-295 (1998)

12. Flueck, M., Hofer, T., Janka, A., Rappaz, J.: Numerical methods for ferromagnetic plates. Scientific report in Analysis and Numerical Analysis, EPFL

13. Munger, D.: Simulation numérique des instabilités magnétohydro-dynamiques dans les cuves de production de l'aluminium. Master's thesis, Université de Montréal (2004)

14. Sele, T.: Instabilities of the metal surface in electrolytic cells. 1, pp. 7-24 (1977)

15. Brezina, M., Mandel, J., Vanek, P.: Convergence of algebraic multigrid based on smoothed aggregation. Numer. Math. 88(3), 559-579 (2001)

16. George, P.-L., Hecht, F., Saltel, E., Borouchaki, H.: GHS3D web site: http://ralyx.inria.fr/2006/Raweb/ gamma/uid31.html. Projet Gamma, INRIA

17. George, P.-L., Hecht, F., Saltel, E.: Fully automatic mesh generator for $3 \mathrm{~d}$ domains of any shape. Impact Comp. Sci. Eng. 2, 187-218 (1990) 